\title{
Modelos Interespecíficos para Controle Químico de Áreas Foliares Lesionadas por Fungos
}

\section{R. ZOTIN ${ }^{1}$, Departamento de Matemática, UNESP, Rio Claro, SP, Brasil}

R.C. BASSANEZI, H.M. YANG, Departamento de Matemática Aplicada, IMECC, UNICAMP, Campinas, SP, Brasil.

\begin{abstract}
Resumo: Apresentamos um modelo para o estudo de uma população de fungos, sujeita a um controle químico, incorporando o efeito da aplicação do fungicida diretamente no modelo e medindo a população de fungos em termos de área lesionada. Os parâmetros, a princípio, são considerados constantes. Posteriormente, são realizadas algumas simulações a título de exemplificação e comparação com o caso constante, considerando a taxa aparente de infecção dependente do tempo (e da temperatura).
\end{abstract}

\section{Introdução}

Os primeiros modelos que surgiram no estudo da dinâmica de fungos na ausência de fungicidas têm como premissa comum o crescimento exponencial de cada subpopulação (sensíveis e resistentes). Além disso, como ocorre com uma grande parte dos modelos matemáticos usados em Ecologia, os parâmetros envolvidos nos modelos são considerados constantes.

Como uma tentativa de incorporar as competições inter e intra específicas, Barrett [2] utilizou o modelo geral de Kolmogorov considerando a dinâmica de duas populações $N_{1}$ e $N_{2}$ que interagem, com taxas de crescimento intrínseco $r_{1}$ e $r_{2}$, respectivamente, dada por

$$
\left\{\begin{array}{lll}
\frac{d N_{1}}{d t} & = & r_{1} N_{1} f_{1}\left(N_{1}, N_{2}\right) \\
\frac{d N_{2}}{d t} & = & r_{2} N_{2} f_{2}\left(N_{1}, N_{2}\right)
\end{array} .\right.
$$

As funções $f_{1}$ e $f_{2}$ descrevem os efeitos das competições inter e intra específicas entre as suas populações.

Os efeitos das competições inter e intra específicas foram incorporados por Varassin [7] numa extensão do modelo proposto por Ghini et al.[4], para o estudo do crescimento de uma população de fungos subdivida em sensíveis $(S)$ e resistentes $(R)$, tomando a seguinte forma :

\footnotetext{
${ }^{1}$ rzotin@rc.unesp.br
} 


$$
\left\{\begin{array}{ll}
\frac{d S}{d t} & =r_{s} S\left[1-\frac{S}{K}-c_{S R} \frac{R}{K}\right]-\alpha r_{s} S\left[1-\frac{S}{K}-c_{S R} \frac{R}{K}\right] \\
\frac{d R}{d t} & =r_{R} R\left[1-\frac{R}{K}-c_{R S} \frac{S}{K}\right]+\alpha r_{s} S\left[1-\frac{S}{K}-c_{S R} \frac{R}{K}\right]
\end{array},\right.
$$

onde

- $K$ é a capacidade suporte, que pode ser dada pelo número de propágulos ou pela área foliar lesionada, dependendo de como a população de fungos está sendo quantificada;

- $r_{S}$ e $r_{R}$ são as taxas aparentes de infecção;

- $c_{S R}$ e $c_{R S}$ medem a intensidade da interação ente $S$ e $R$;

- o termo $\alpha r_{s} S\left[1-S / K-c_{S R} R / K\right]$ representa o fluxo de sensíveis para resistentes, onde $\alpha$ é a frequência de mudança.

Este modelo é, portanto, um caso particular do modelo de Lotka-Volterra e uma análise dos pontos de equilíbrio e estabilidade também é apresentada em [7].

Neste trabalho propomos uma extensão do modelo descrito pelas equações (1.2) incorporando o efeito da aplicação do fungicida diretamente no modelo e medindo a população de fungos em termos de área lesionada.

A partir disso, pretendemos obter uma estratégia de controle ótima no sentido de minimizar tanto o fungicida aplicado quanto a área lesionada pela população de fungos durante o intervalo de tempo decorrido entre o momento em que a doença é detectada $(t=0)$ e o momento da colheita $\left(t=t_{f}\right)$.

\section{Modelo: Controle de Área Lesionada (r Cons- tante)}

O modelo que propomos para o controle de fungos com aplicação de fungicidas em populações que desenvolvem resistência está sujeito às seguintes hipóteses:

a) A "população" de fungos (representada pela área total ocupada $N$ ) analisada em uma certa cultura é subdividida em sensíveis e resistentes, sendo que $S(t)$ e $R(t)$ representam a fração de área foliar da cultura lesionada por sensíveis e resistentes, respectivamente, em cada instante de tempo. Inicialmente, antes da aplicação de fungicida, temos as condições iniciais dadas por $S(0)=S_{0}>0$ e $R(0)=R_{0}>0$;

b) as taxas aparentes de infecção provocadas por sensíveis e resistentes são $r_{s}$ e $r_{r}$, respectivamente;

c) a taxa de mudança de sensível para resistente é dada por $\alpha$;

d) a eficácia do fungicida aplicado é dada por $\beta$ e incorpora o fato da mesma depender da concentração do fungicida utilizado; 
e) a taxa de aplicação de fungicida é dada por $u=u(t)$, função contínua por partes. Além disso, utilizamos uma limitação nessa aplicação da forma $0 \leq u(t) \leq 1$, ou seja, consideramos u sempre em relação a um valor $u_{\max }$ permitido;

f) consideramos também que $d S / d t>0$ e $d R / d t>0$ no sentido de, uma vez que uma folha é atacada por fungos, a área lesionada não mais se regenerar mesmo com a aplicação de fungicida.

Com estas considerações formulamos o seguinte modelo matemático:

$$
\left\{\begin{array}{l}
\dot{S}(t)=(1-\alpha) r_{s} S(1-S-R)(1-\beta u) \\
\dot{R}(t)=r_{r} R(1-S-R)+\alpha r_{s} S(1-S-R)(1-\beta u) \\
S(0)=S_{0}, R(0)=R_{0}, \quad 0 \leq u(t) \leq 1
\end{array}\right.
$$

Embora $S, R, N$ e $u$ sejam funções de $t$, estaremos omitindo a variável $t$ para simplificarmos a notação.

Tratanto o sistema (2.1) em termos da área total ocupada e considerando $r_{s}=$ $r_{r}=r$, o sistema (2.1) pode ser escrito como:

$$
\left\{\begin{array}{l}
\dot{N}(t)=r N(1-N)(1-\beta u)+r R \beta u(1-N) \\
\dot{R}(t)=r R(1-N)+\alpha r(N-R)(1-N)(1-\beta u) \\
N(0)=N_{0}=S_{0}+R_{0}, \quad 0 \leq u(t) \leq 1
\end{array}\right.
$$

O problema de controle ótimo associado à dinâmica (2.2) consiste em encontrar $u^{*}(t)$ que minimiza o funcional

$$
J(u)=N\left(t_{f}\right)+c_{1} \int_{0}^{t_{f}} u(t) d t,
$$

ou seja, $J\left(u^{*}\right)=\min _{u} N\left(t_{f}\right)+c_{1} \int_{0}^{t_{f}} u(t) d t$.

Consideramos o tempo final $\left(t_{f}\right)$ fixo, que corresponde ao dia da colheita ou ao tempo máximo em que é permitida a aplicação do fungicida..

Introduzindo o Hamiltoniano [5] associado a este problema (omitindo a variável $t$ de $\lambda_{1}$ e $\left.\lambda_{2}\right)$, temos

$$
\begin{aligned}
H\left(t, u, \lambda_{1}, \lambda_{2}, S, R\right)= & c_{1} u+\lambda_{1}[r N(1-N)(1-\beta u)+r R \beta u(1-N)] \\
& +\lambda_{2}[r R(1-N)+\alpha r(N-R)(1-N)(1-\beta u)] .
\end{aligned}
$$

As equações adjuntas (ou de coestado), em função do Hamiltoniano, são dadas por:

$$
\dot{\lambda}_{1}^{*}=-\partial H / \partial N \quad \text { e } \quad \dot{\lambda}_{2}^{*}=-\partial H / \partial R
$$


que, na sua forma já simplificada, são descritas por:

$$
\left\{\begin{array}{l}
\dot{\lambda}_{1}^{*}=-\left[r\left(1-\beta u^{*}\right)\left(1-2 N^{*}\right)-r \beta R^{*} u^{*}\right] \lambda_{1}^{*}+r R^{*} \lambda_{2}^{*} \\
-\lambda_{2}^{*} \alpha r\left(1-\beta u^{*}\right)\left(1-2 N^{*}+R^{*}\right),
\end{array} \quad \begin{array}{l}
\dot{\lambda}_{2}^{*}=-r\left(1-N^{*}\right)\left[1-\alpha\left(1-\beta u^{*}\right)\right] \lambda_{2}^{*}-\lambda_{1}^{*} r \beta u^{*}\left(1-N^{*}\right), \\
\operatorname{com} \quad \lambda_{1}^{*}\left(t_{f}\right)=1 \quad \text { e } \quad \lambda_{2}^{*}\left(t_{f}\right)=0 .
\end{array}\right.
$$

As condições finais sobre $\lambda_{1}^{*}$ e $\lambda_{2}^{*}$ vêm do fato de $t_{f}$ ser fixo e $N^{*}\left(t_{f}\right)$ e $R^{*}\left(t_{f}\right)$ serem livres.

Voltando ao Hamiltoniano dado por (2.4) e simplificando as expressões, obtemos

$$
\begin{aligned}
H\left(t, u, \lambda_{1}, \lambda_{2}, S, R\right)= & {\left[c_{1}-r(1-N)(N-R) \beta\left(\lambda_{1}+\alpha \lambda_{2}\right)\right] u } \\
& +\lambda_{1} r N(1-N)+\lambda_{2} r R(1-N) \\
& +\alpha r \lambda_{2}(N-R)(1-N) .
\end{aligned}
$$

Assim, denotando a função que decide o controle por $g(t)$, vem

$$
g(t)=c_{1}-r(1-N)(N-R) \beta\left(\lambda_{1}+\alpha \lambda_{2}\right),
$$

e, portanto, $u^{*}(t)$ é dado por

$$
u^{*}(t)=\left\{\begin{array}{cl}
0, & \text { se } g(t)>0 \\
1, & \text { se } g(t)<0 \\
\text { indet., } & \text { se } g(t)=0
\end{array}\right.
$$

A não linearidade do modelo torna as equações diferenciais para $\lambda_{1}$ e $\lambda_{2}$ complicadas do ponto de vista analítico, tornando-se praticamente impossível uma análise através das expressões para $\lambda_{1}$ e $\lambda_{2}$. Consequentemente, a análise da função $g(t)$ para a obtenção da lei de controle ótimo não é imediata.

Optamos, assim, por "investigar" quais tipos de controles são factíveis através de demonstrações por absurdo, ou seja, supõe-se que o controle tem um determinado aspecto e chega-se a uma contradição caso ele são seja factível.

Lema 1 Se o controle ótimo for zero $\left(u^{*}(t)=0\right)$ durante algum intervalo $\left(\bar{t}, t_{f}\right]$, então $u^{*}(t)=0 \forall t \in\left[0, t_{f}\right]$, considerando $\alpha \approx 0$.

Demonstração: Suponha que o controle seja da forma

$$
u^{*}(t)= \begin{cases}1, & 0 \leq t<\bar{t} \\ 0, & \bar{t}<t \leq t_{f}\end{cases}
$$

ou seja, o controle acaba com zero e tem uma única troca em $\bar{t}(g(\bar{t})=0)$.

Como $u^{*}(t)=0$ para $t \in\left(\bar{t}, t_{f}\right]$ temos $g(t)>0$ neste intervalo, e as equações diferenciais (2.5) para $\lambda_{1}^{*}$ e $\lambda_{2}^{*}$ tomam a seguinte forma:

$$
\dot{\lambda}_{2}^{*}+r\left(1-N^{*}\right)(1-\alpha) \lambda_{2}^{*}=0
$$




$$
\dot{\lambda}_{1}^{*}+r\left(1-2 N^{*}\right) \lambda_{1}^{*}=-\lambda_{2}^{*}\left[-r R^{*}+\alpha r\left(1-2 N^{*}+R^{*}\right)\right] .
$$

Usando o fato de conhecermos $\lambda_{2}^{*}$ em $t=t_{f}$, obtemos como solução de (2.10)

$$
\lambda_{2}^{*}(t)=\lambda_{2}^{*}\left(t_{f}\right) e^{\int_{t}^{t_{f}} r(1-\alpha)(1-N) d s}=0, \quad \forall t \in\left(\bar{t}, t_{f}\right],
$$

pois $\lambda_{2}^{*}\left(t_{f}\right)=0$.

Consequentemente, temos como solução para $\lambda_{1}^{*}$, usando o fato de $\lambda_{1}^{*}\left(t_{f}\right)=1$

$$
\lambda_{1}^{*}(t)=e^{\int_{t}^{t_{f}} r\left(1-2 N^{*}\right) d s}>0, \quad \forall t \in\left(\bar{t}, t_{f}\right] .
$$

Analisando o comportamento de $g(t)$, a função que decide o controle, temos:

$$
g^{\prime}(t)=r^{2} \beta\left(1-N^{*}\right)\left(N^{*}-R^{*}\right)\left[\alpha \lambda_{1}^{*}\left(1-N^{*}\right)+\lambda_{2}^{*}\left[(\alpha-1) R^{*}+\alpha\left(1-N^{*}\right)\right]\right] .
$$

Tomando $\alpha \approx 0$ (na prática está no intervalo $10^{-9}<\alpha<10^{-5}$ ) e $\lambda_{2}^{*}=0$, temos $g^{\prime}(t)=0 \forall \mathrm{t} \in\left(\bar{t}, t_{f}\right] \Rightarrow g(t)=$ constante $>0, \forall \mathrm{t} \in\left(\bar{t}, t_{f}\right]$. Pela continuidade de $g(t)$, não é possível $g(\bar{t})=0$, ou seja, $\bar{t}$ suposto inicialmente não existe. Assim, $u^{*}(t)=0, \forall \mathrm{t} \in\left[0, t_{f}\right]$.

Do lema anterior, a possibilidade de que, a partir de um certo instante, $u^{*}(t)$ tenha o comportamento descrito em (2.9) é excluída. Assim, o controle ótimo, se não for nulo sempre, deve ser tal que acabe com $u^{*}(t)=1$.

Analisaremos agora uma outra situação.

Lema 2 Se o controle ótimo terminar com $u^{*}(t)=1$ e $\alpha \approx 0$, então ele terá no máximo uma troca de controle.

Demonstração: Suponha que o controle seja da forma

$$
u^{*}(t)=\left\{\begin{array}{cc}
1 ; & 0 \leq t<\bar{t}_{1} \\
0 ; & \bar{t}_{1}<t<\bar{t}_{2} \\
1 ; & \bar{t}_{2}<t \leq t_{f}
\end{array}\right.
$$

$\mathrm{e}$, consequentemente, teremos

$$
g(t)\left\{\begin{array}{cc}
<0 ; & 0 \leq t<\bar{t}_{1} \\
>0 ; & \bar{t}_{1}<t<\bar{t}_{2} . \\
<0 ; & \bar{t}_{2}<t \leq t_{f}
\end{array}\right.
$$

Considerando novamente $\alpha \approx 0$, vamos analisar o comportamento de $g(t)$ em cada subintervalo:

- Para $0 \leq t<\bar{t}_{1}$ temos $g(t)<0$ e, portanto, $g(t)$ deve ser crescente para que exista troca de controle em $t=\bar{t}_{1}$. Assim, $g^{\prime}(t)>0 \Rightarrow \lambda_{2}^{*}(t)<0$; 
- $\operatorname{Em} \bar{t}_{1}<t<\bar{t}_{2}$ as equações diferenciais para $\lambda_{1}^{*}$ e $\lambda_{2}^{*}$ são:

$$
\left\{\begin{array}{l}
\dot{\lambda}_{1}^{*}+r\left(1-2 N^{*}\right) \lambda_{1}^{*}=r R \lambda_{2}^{*}, \\
\dot{\lambda}_{2}^{*}+r\left(1-N^{*}\right) \lambda_{2}^{*}=0,
\end{array}\right.
$$

cuja solução para $\lambda_{2}^{*}$ é

$$
\lambda_{2}^{*}(t)=\lambda_{2}^{*}\left(\bar{t}_{1}\right) \exp \int_{\bar{t}_{1}}^{t}-r\left(1-N^{*}\right) d s .
$$

Como $g(t)>0$ para $\bar{t}_{1}<t<\bar{t}_{2}$ e $g(t)<0$ em $\bar{t}_{2}<t \leq t_{f}, g(t)$ deve continuar crescendo para $t>\bar{t}_{1}$, atingir um máximo em $t=\tilde{t}$ dentro desse intervalo para que, posteriormente, $g\left(\bar{t}_{2}\right)=0$.

Dessa forma, $\lambda_{2}^{*}(t)<0$ em $\bar{t}_{1}<t<\tilde{t}, \lambda_{2}^{*}(\tilde{t})=0$ e $\lambda_{2}^{*}(t)>0$ para $\left(\tilde{t}, t_{f}\right]$.

Voltando em (2.11) concluimos que $\lambda_{2}^{*}(t)<0$ em todo o intervalo $\left(\bar{t}_{1}, \bar{t}_{2}\right)$, o que nos leva a uma contradição.

Com a exclusão de alguns possíveis controles, através dos dois lemas anteriores podemos enunciar um resultado geral:

Teorema 1 Para o problema de controle (2.2)-(2.3), com $\alpha \approx 0$, o controle ótimo $u^{*}(t)$ é do tipo

$$
u^{*}(t)=\left\{\begin{array}{cc}
0, & 0 \leq t<\bar{t} \\
1, & \bar{t}<t \leq t_{f}
\end{array}\right.
$$

$\operatorname{com} \bar{t} \in\left[0, t_{f}\right]$.

Demonstração: Ver lemas 1 e 2.

Do teorema anterior conseguimos identificar quais os possíveis tipos de controle que, naturalmente, dependem dos parâmetros envolvidos no modelo: $S_{0}, R_{0}, \alpha, \beta$, $c_{1}$ e $r$. No entanto, para este modelo estudado não obtivemos uma relação explícita entre os parâmetros de maneira a identificar, por exemplo, quando $u^{*}(t)=0, \forall t \in$ $\left[0, t_{f}\right]$.

\subsection{Aplicação: controle de fungos do feijoeiro}

No trabalho de Bassanezi et al.[3] alguns parâmetros monocíclicos do fungo causador da ferrugem (Uromyces appendiculatus) e da mancha angular (Phaeoisariopsis griseola) no feijoeiro foram quantificados a diferentes temperaturas em dois cultivares: Rosinha G-2 e Carioca. Na descrição do procedimento realizado para a análise dos dados, observa-se que o desenvolvimento de cada tipo de fungo é analisado através de área lesionada e não com o número de propágulos. Outros parâmetros foram observados como, por exemplo, a severidade da doença (\% de área foliar com lesões) e a taxa de crescimento das lesões. 
Os resultados obtidos para este último parâmetro podem ser visualizados na Figura 1, observando-se que a temperatura ótima para o desenvolvimento da ferrugem é de $17^{\circ} \mathrm{C}$ e para a mancha angular, $24^{\circ} \mathrm{C}$.

Utilizamos os dados da Figura 1 nas simulações realizadas e procuramos observar a variação do controle quando alteramos cada um dos parâmetros, já que uma relação explícita entre $\bar{t}$ e os demais parâmetros presentes no modelo não foi obtida.

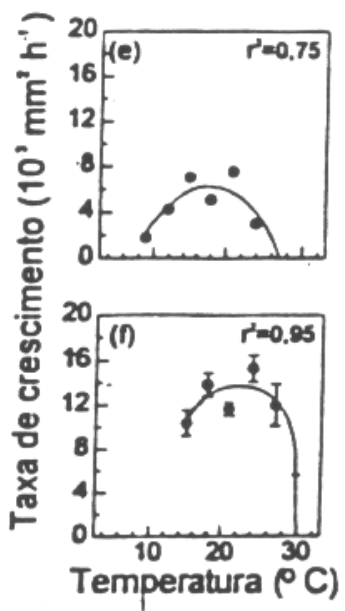

(a)

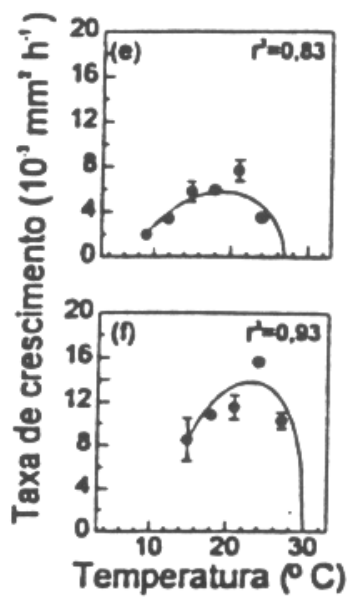

(b)

Figura 1: (a) taxa de crescimento do fungo causador da ferrugem e da mancha angular, respectivamente, ao cultivar Rosinha G-2, em função da temperatura. (b) análogo ao item anterior para cultivar Carioca [3].

Nas simulações realizadas os parâmetros foram utilizados com as seguintes unidades: $\alpha$ (adimensional), $t, t_{f}$ e $\bar{t}$ (dias), $r$ e $\beta$ (dias ${ }^{-1}$ ), $c_{1}$ (área lesionada/dias). Por questões de simplificação, as unidades serão omitidas na descrição das simulações assim como na maioria dos gráficos. Utilizamos o software desenvolvido por Adami [1], em linguagem Fortran, que tem por objetivo resolver problemas de Controle Ótimo utilizando um algoritmo conhecido como BOX, de programação não linear. Neste algoritmo o objetivo é a resolução de um problema do tipo

$$
\begin{gathered}
\min f(x) \\
\text { s.a } \mathrm{x}_{\min } \leq x \leq x_{\max }
\end{gathered}
$$

onde $f: R^{n} \rightarrow R$ é diferenciável e $x_{\min }, x_{\max } \in \mathrm{R}^{n}$. As tabelas e os gráficos foram realizados utilizando o software Excel.

Vamos considerar que, na cultura analisada, a fração total de área infectada é de $10 \%(N(0)=0,1)$, onde $S(0)=0,08$ e $R(0)=0,02(t=0$ é o instante em que a 
doença é observada) e que a doença foi detectada restando 80 dias para a colheita, ou seja, $t_{f}=80$ dias. Vejamos algumas situações analisadas:

a) Influência de $r$ na determinação de $\bar{t}$ (instante de comutação do tratamento):

Consideramos fixos os parâmetros $\beta, c_{1}, \alpha$, as condições iniciais $S_{0}, R_{0}$ e realizamos algumas simulações utilizando alguns valores de $r$ obtidos em [3] para a mancha angular na cultivar Rosinha G-2. Dentre os valores de $r$, escolhemos: $r_{1}=0,026 \mathrm{~A} /$ dia onde $A=10 \mathrm{~mm}^{2}, r_{2}=0,031 \mathrm{~A} /$ dia e $r_{3}=0,0336 \mathrm{~A} /$ dia. Embora neste experimento realizado a taxa de crescimento máxima obtida seja $r_{3}$, consideraremos diversos valores de $r$ (maiores que $r_{3}$ e também menores que $r_{1}$ ) com o propósito de observarmos a variação de $\bar{t}$ em função de $r$.

Na tabela que será apresentada a seguir podemos observar a variação de $\bar{t}$ em função de $r$ assim como a área total lesionada $\left(S^{*}\left(t_{f}\right), R^{*}\left(t_{f}\right), N^{*}\left(t_{f}\right)\right)$ após o respectivo controle efetuado, sendo que, do Teorema 1 , se existe troca de controle ela ocorre de $u=0$ para $u=1$. Para $\bar{t}=80$ temos $u^{*}(t)=0, \forall t \in[0,80]$ e, para $\bar{t}=0$, temos $u^{*}(t)=1$.

\begin{tabular}{|c|c|c|c|c|}
\hline$r$ & $t$ & $S^{\star}(t f)$ & $R^{\star}(t f)$ & $N^{\star}(t f)$ \\
\hline 0,01 & 80 & 0,1586 & 0,0396 & 0,1982 \\
\hline 0,015 & 80 & 0,2155 & 0,05389 & 0,26939 \\
\hline 0,016 & 80 & 0,2284 & 0,0571 & 0,2855 \\
\hline 0,017 & 80 & 0,2416 & 0,0604 & 0,302 \\
\hline 0,018 & 80 & 0,2553 & 0,0638 & 0,3191 \\
\hline 0,019 & 79,19 & 0,261 & 0,0674 & 0,3284 \\
\hline 0,02 & 61,5 & 0,2437 & 0,07152 & 0,31522 \\
\hline 0,021 & 49,24 & 0,23 & 0,0762 & 0,3062 \\
\hline 0,022 & 38,9 & 0,2191 & 0,0815 & 0,3006 \\
\hline 0,023 & 30,75 & 0,2099 & 0,0872 & 0,2971 \\
\hline 0,024 & 24,72 & 0,2022 & 0,0935 & 0,2957 \\
\hline 0,025 & 18,49 & 0,1961 & 0,1 & 0,2961 \\
\hline 0,026 & 14,27 & 0,1906 & 0,1072 & 0,2978 \\
\hline 0,027 & 10,25 & 0,1865 & 0,1147 & 0,3012 \\
\hline 0,028 & 6,23 & 0,1833 & 0,1225 & 0,3058 \\
\hline 0,029 & 4,22 & 0,1804 & 0,1309 & 0,3113 \\
\hline 0,03 & 2,21 & 0,1784 & 0,1396 & 0,318 \\
\hline 0,031 & 0 & 0,1783 & 0,1483 & 0,3266 \\
\hline 0,032 & 0 & 0,1821 & 0,1564 & 0,3385 \\
\hline 0,036 & 0 & 0,1976 & 0,1918 & 0,3894 \\
\hline 0,04 & 0 & 0,2129 & 0,2312 & 0,4441 \\
\hline 0,045 & 0 & 0,2314 & 0,2846 & 0,516 \\
\hline 0,05 & 4,22 & 0,2664 & 0,3285 & 0,5949 \\
\hline 0,055 & 9,24 & 0,3271 & 0,3478 & 0,6749 \\
\hline 0,056 & 10,25 & 0,3374 & 0,3516 & 0,689 \\
\hline 0,057 & 11,25 & 0,3518 & 0,3521 & 0,7039 \\
\hline 0,058 & 12,26 & 0,3637 & 0,3539 & 0,7176 \\
\hline 0,059 & 13,26 & 0,378 & 0,3534 & 0,7314 \\
\hline 0,06 & 14,17 & 0,3899 & 0,3542 & 0,7441 \\
\hline 0,065 & 18,49 & 0,4602 & 0,3433 & 0,8035 \\
\hline 0,07 & 22,71 & 0,5293 & 0,3227 & 0,852 \\
\hline 0,075 & 80 & 0,7825 & 0,1956 & 0,9781 \\
\hline 0,077 & 80 & 0,785 & 0,1962 & 0,9812 \\
\hline 0,08 & 80 & 0,7881 & 0,197 & 0,9851 \\
\hline
\end{tabular}

Tabela 1 - Valores de $\bar{t}$ e $\left(S^{*}\left(t_{f}\right), R^{*}\left(t_{f}\right), N^{*}\left(t_{f}\right)\right)$ para cada taxa de crescimento $r$ dada, considerando os parâmetros e as condições iniciais fixos: $S_{0}=0,08, R_{0}=0,02$, $c_{1}=0,002$ e $\beta=0,6$. 
Na Tabela 1 tomamos $S_{0}=0,08, R_{0}=0,02, c_{1}=0,002, \beta=0,6$ e $\alpha=10^{-5}$. Podemos observar que, com a eficiência $\beta$ e "custo" $c_{1}$ fixados, à medida que $r$ aumenta passa a existir troca de controle de modo que o período de aplicação aumenta ( $\bar{t}$ diminui) até que, para $r \in[0.031,0.045]$ torna-se necessário a aplicação durante todo o intervalo $[0,80]$. Para valores de $\mathrm{r}$ maiores que 0,045 o valor de $\bar{t}$ aumenta e a aplicação de fungicida torna-se inviável para valores de $\mathrm{r}$ muito altos (veja Figura 2(a)). Na Figura 2(b) observamos o comportamento da área lesionada no tempo final $t_{f}$ para cada tipo de controle obtido (em função de r).

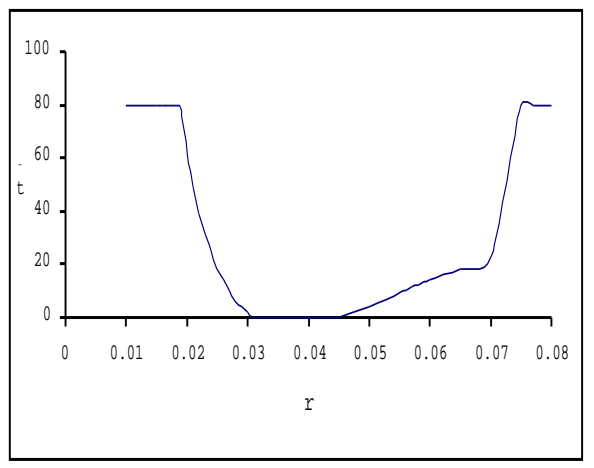

(a)

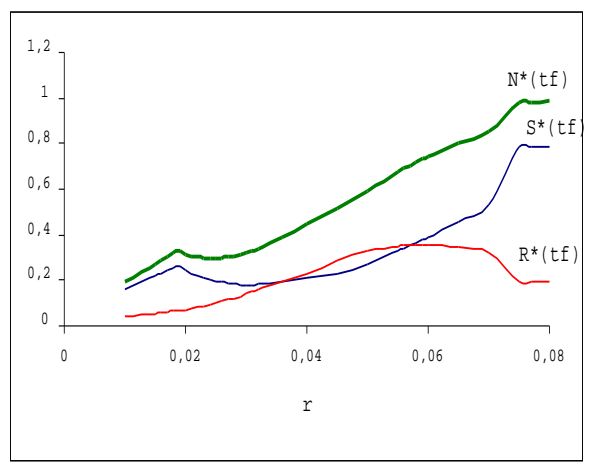

(b)

Figura 2 - (a) Instante de comutação $\bar{t}$ do controle em função da taxa de crescimento $r$ do fungo (dados fixados na Tabela 1). (b) $S^{*}\left(t_{f}\right), R^{*}\left(t_{f}\right)$ e $N^{*}\left(t_{f}\right)$ em função de $r$ para os dados da Tabela $1 . N^{*}\left(t_{f}\right)$ é a percentagem de área lesionada no tempo final $t_{f}$ para cada valor de $r$ fixo.

Mantendo os mesmos parâmetros da Tabela 1 , exceto $c_{1}$ e $\beta$, que foram considerados como $c_{1}=0,004$ e $\beta=0,8$, não temos o mesmo tipo de comportamento já observado. Para este novo conjunto de dados não observamos um intervalo de $\mathrm{r}$ para o qual $u^{*}(t)=1$ (como na Tabela 1 ) mas sim um intervalo de $r$ onde a troca de controle praticamente não sofre alteração.

Se tomarmos os mesmos parâmetros da Tabela 1, porém com $c_{1}=0,02(10$ vezes maior que o utilizado) encontramos que $u^{*}(t)=0$ sempre, para qualquer valor de $r$ da tabela.

\section{b) Influência de $\beta$ e $c_{1}$ na determinação de $\bar{t}$ :}

Mantendo fixos $\alpha=10^{-5}, S_{0}=0,08, R_{0}=0,02, r=0,026, t_{f}=80$ e variando os parâmetros $\beta$ e $c_{1}$, observamos na tabela a seguir o valor de $\bar{t}$ obtido. Para este conjunto de dados tem-se que, fixando $\beta$, existe um intervalo $[a, b]$ de $c_{1}$ para o qual existe troca de controle e, à medida que $c_{1}$ aumenta $\left(c_{1}>b\right)$ torna-se inviável a aplicação de fungicida; se $c_{1}<a$ então $u^{*}(t)=1(\bar{t}=0), \forall t \in[0,80]$. 


\begin{tabular}{|c|c|c|c|c|c|c|c|c|c|}
\hline$\beta \backslash^{c_{1}}$ & 0,0015 & 0,00175 & 0,002 & 0,00225 & 0,0025 & 0,003 & 0,0035 & 0,004 & 0,0045 \\
\hline 0,8 & 0 & 3,21 & 10,25 & 18,49 & 24,72 & 38,99 & 53,26 & 71,75 & 80 \\
\hline 0,75 & 0 & 2,21 & 10,25 & 18,49 & 26,73 & 42,2 & 59,49 & 80 & 80 \\
\hline 0,7 & 0 & 2,21 & 10,25 & 20,5 & 28,94 & 47,43 & 68,73 & 80 & 80 \\
\hline 0,65 & 0 & 2,21 & 12,26 & 22,71 & 32,76 & 55,27 & 80 & 80 & 80 \\
\hline 0,6 & 0 & 2,21 & 14,27 & 26,73 & 38,99 & 68,73 & 80 & 80 & 80 \\
\hline
\end{tabular}

Tabela 2 - Valor de $\bar{t}$ para cada par $\left(\beta, c_{1}\right)$ - Por exemplo, para $c_{1}=0,003$ e $\beta=0,7$ tem-se que $\bar{t}=47,43$.

Embora não tenhamos uma relação explícita entre os parâmetros para a obtenção de $u^{*}(t)$, as simulações numéricas utilizando o pacote desenvolvido em [1] possibilitou-nos alguma análise e obtenção de $u^{*}(t)$.

\section{Taxa de Crescimento Dependente do Tempo}

No caso específico de fungos, vários fatores exercem grande influência no seu desenvolvimento como umidade, luminosidade, temperatura, dentre outros. No que se refere à temperatura, a atividade fúngica começa a uma temperatura mínima, aumenta até um valor ótimo e então declina e pára a uma temperatura máxima. Estas temperaturas são conhecidas como temperaturas cardinais e dependem de outros fatores, que inclui o tempo de exposição a qualquer temperatura [6]. Além disso, para cada tipo de fungo e sob diferentes condições, estas temperaturas cardinais podem variar sensivelmente.

Diante dessas considerações a respeito da dependência da taxa de crescimento em relação a temperatura (e consequentemente do tempo), efetuaremos algumas simulações a título de comparação com o caso constante, tomando $r(t)$ como uma função periódica.

Utilizaremos alguns dos dados presentes na Figura 1 para a taxa de crescimento da lesão da mancha angular ao cultivar Rosinha G-2. Por exemplo, a temperatura ótima para o desenvolvimento desse fungo $\left(24^{\circ} \mathrm{C}\right)$ e os valores de $r$ nas temperaturas $24^{\circ} \mathrm{C}\left(r=0,014 \mathrm{~mm}^{2} / h\right)$ e $29^{\circ} \mathrm{C}\left(r=0,002 \mathrm{~mm}^{2} / h\right)$. Esses valores de $r$ serão utilizados com as mesmas unidades já usadas nas simulações anteriores.

Se considerarmos que a temperatura máxima atingida durante o ano é $39^{\circ} \mathrm{C}$ e que a mínima é $1^{\circ} C$, podemos simular a temperatura em função de $t$ (dias do ano, de janeiro a dezembro) da seguinte forma:

$$
T(t)=20+19 \cos (\pi t / 180),
$$

onde $20^{\circ} \mathrm{C}$ corresponde a uma temperatura média anual.

Utilizando a idéia de temperaturas cardinais citadas anteriormente, ou seja, a atividade dos fungos começa a uma temperatura mínima, aumenta até um ótimo e então declina, vamos supor a taxa de crescimento em função da temperatura da forma:

$$
r(T)=a \exp \left[-b\left(T-T^{*}\right)\right]
$$


onde $T^{*}$ é a temperatura em que a maior taxa de crescimento é atingida e os coeficientes $a$ e $b$ podem ser determinados a partir de informações de $r$ em algumas temperaturas.

Como esse fungo praticamente não se desenvolve em temperaturas inferiores a $17^{\circ} \mathrm{C}$ e superiores a $30^{\circ} \mathrm{C}$, para efeito de simplificação e obtenção de $r(t)$ de modo contínuo, vamos considerar

$$
\begin{gathered}
T=24^{\circ} C \quad \Rightarrow r=0,0336 \\
T=6^{\circ} C \quad \Rightarrow r=0,0048
\end{gathered}
$$

Assim, obtemos a função $r(t)=r(T(t))$, dada por

$$
r(t)=0,0336 \exp \left[-0,006 *(-4+19 * \cos (\pi t / 180))^{2}\right],
$$

cujo gráfico pode ser visualizado a seguir:

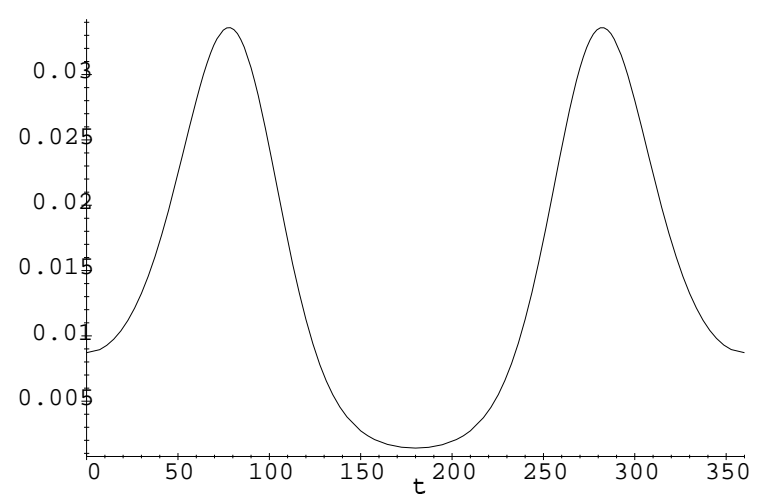

Figura 3 - Gráfico de $r(t)=0,0336 \exp \left[-0,006 *(-4+19 * \cos (\pi t / 180))^{2}\right]$.

Utilizando $S_{0}=0,08, R_{0}=0,02, \alpha=10^{-5}, \beta=0,6, c_{1}=0,002$ e a função $r(t)$ acima, observamos que o controle obtido não necessariamente apresenta o mesmo tipo de comportamento do caso $r$ constante.

Tomando $r$ para $t \in[50,130]$ obtém-se uma troca de controle em $\bar{t}_{1}=4,22 \mathrm{e}$ $\bar{t}_{2}=50,24$ enquanto que, considerando $t \in[0,80]$ obtemos $\bar{t}=52,25$. Nas figuras que se seguem podemos observar estes resultados e comparar $r(t)$ com o intervalo de tempo em que $u^{*}(t)=1$, ou seja, $u^{*}(t)=1$ para uma "faixa" em que $r(t)$ é máxima. 

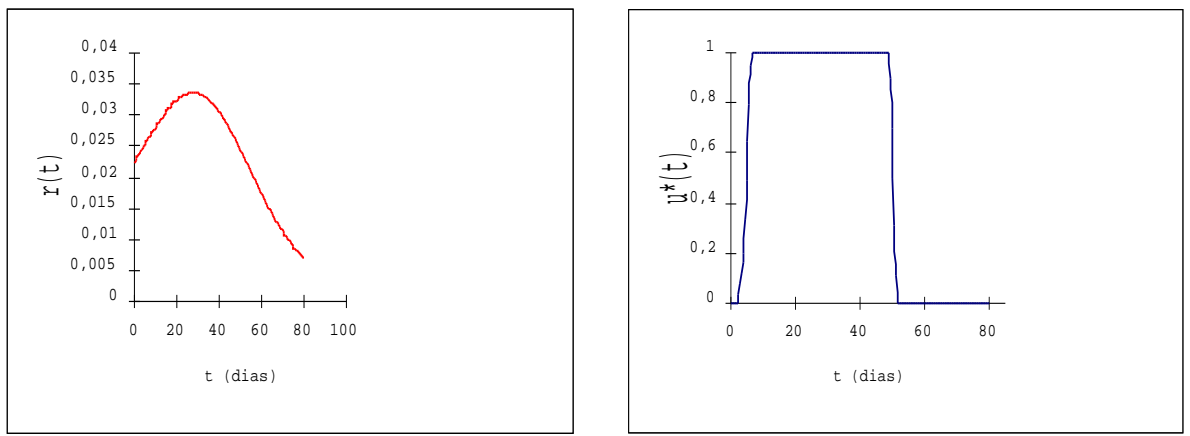

(a)
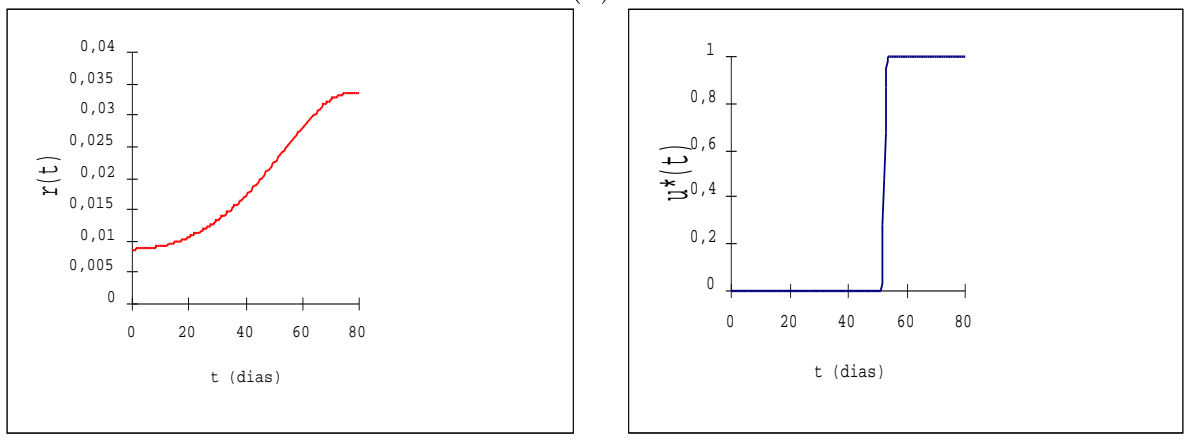

(b)

Figura 4 - Intervalo da função $r(t)$ considerado e o respectivo controle obtido - (a) $r$ para $t \in[50,130]$, (b) $r$ para $t \in[0,80]$.

Vale a pena ressaltar que a passagem de $u=0$ para $u=1$, utilizando métodos numéricos, não ocorre de forma instantânea, o que pode ser observado nos gráficos para $u^{*}(t)$.

Assim, a inclusão do efeito da temperatura na taxa de crescimento das lesões resulta em um comportamento diferente em modelo linear [8] e em modelo não linear.

Embora esse fato só tenha sido observado numericamente sem uma demonstração formal, constatamos que a não linearidade do modelo, com a inclusão das competições inter e intra específicas, tem uma grande influência na determinação de uma estratégia ótima de controle, principalmente quando consideramos $r=r(t)$ variável.

\section{Considerações Finais}

No caso específico deste trabalho buscamos modelar matematicamente uma situação de produção agrícola sujeita ao ataque de fungos, onde se buscava otimizar a produção (minimizando a infestação no final da colheita) com um custo menor 
possível no combate à doença (otimizando o uso de agrotóxico) considerando "populações de fungos" sensíveis e resistentes aos fungicidas.

Consideramos a dinâmica da área foliar lesionada contemplando a competição intra-específica entre as populações de fungos sensíveis e resistentes pela ocupação da área foliar. Tal ocupação é cumulativa, isto é, uma vez lesionada a área foliar não se recupera para a fotossíntese, o que acarrreta uma menor produtividade da planta. A produtividade é afetada significativamente se a região lesada ultrapassa algum índice de infestação que varia para cada cultivar. O problema de controle que procuramos analisar visou estabelecer um valor mínimo para a região foliar lesionada no tempo final (colheita) sujeita ao gasto (aplicação) mínimo de fungicidas.

Consideramos $r$ constante e $r=r(t)$ variável com o tempo sendo que no primeiro caso a troca de controle (quando existe) é de $u=0$ para $u=u_{\max }$ e, para $r(t)$ variável, existe a possibilidade de duas trocas de controle (embora só observado em simulações) sendo que $u=u_{\max }$ é obtido sempre no intervalo onde a taxa de crescimento é máxima. Comportamento semelhante não foi observado para o modelo linear em [8], que possui no máximo uma troca de controle, tanto para $r=$ constante como para $r=r(t)$.

É importante ressaltarmos que, tanto no modelo linear tratado em [8] como no modelo não linear aqui proposto, obtivemos algumas particularidades de cada modelo apresentado considerando $\alpha \approx 0$. Assim, a mutação de sensível para resistente tem pouca influência na adoção de uma medida de controle quando desejamos identificar como o fungicida deve ser aplicado, logo após a doença ter sido detectada. A pressão de seleção se mostra muito mais importante para o desenvolvimento da resistência do que as mutações, uma vez que a resistência está sempre presente na população. Neste caso, não estamos levando em consideração aplicações anteriores, mas o nível atingido pela "população" resistente, logo após o controle, pode ser analisado para futuras aplicações.

\section{Referências}

[1] A. Adami, Resolução de problemas de controle ótimo utilizando o algoritmo BOX - Tese de Mestrado, IMECC- UNICAMP, 1996.

[2] J.A. Barrett, Estimating Relative Fitness in Plant Parasites: Some General Problems, Phytopathology, 73 No.4 (1983).

[3] R.B. Bassanezi, L. Amorin, A.B. Filho e C.V. Godoy, Análise Comparativa entre a ferrugem e a mancha angular do feijoeiro: efeito da temperatura nos parâmetros monocíclicos, Fitopatologia brasileira, 22 No.3 (1997).

[4] R. Ghini, L.L. Vendite e A.A. Petrucci, Dosagens de fungicidas: avaliação de seus efeitos na resistência de fungos através de um modelo matemático, Fitopat. Bras., 19 (1994), 514-519.

[5] D. Kirk, "Optimal Control Theory- An Introduction", Prentice Hall, 1970. 
[6] C.S. Lacaz, P.S. Minami, A. Purchio, "O grande mundo dos Fungos", Editora Polígono - Universidade de São Paulo, 1970.

[7] C.G. Varassin, Modelagem Matemática para o crescimento de populações de fungos e o desenvolvimento da resistência a fungicidas, Tese de Mestrado, IMECC - UNICAMP, 1996.

[8] R. Zotin, Controle Ótimo da Aplicação de Fungicidas na Lavoura, "Tese de Doutorado", UNICAMP, 1999. 